\title{
Yellow Nail Syndrome Associated With Rheumatoid Arthritis
}

Yoshinori Taniguchi $\mathbb{D}, \mathrm{MD}$, Department of Endocrinology, Metabolism, Nephrology and Rheumatology, Kochi Medical School Hospital, Nankoku, and Department of Rheumatology, Bay Side Misato Medical Center, Kochi, Japan; Takahito Kimata, MD, Department of Rheumatology, Bay Side Misato Medical Center, Kochi, Japan. Address correspondence to Dr. Y. Taniguchi, Department of Endocrinology, Metabolism, Nephrology and Rheumatology, Kochi Medical School Hospital, Kochi University, Kohasu, Oko-cho, Nankoku, Kochi 783-8505, Japan. Email: yoshii.tan@gmail.com. Ethics board approval is not required because this is a single case report and no intervention had been made for research. We obtained the patient's written informed consent to publish the material.

Yellowish-white nails have been known to be associated with various malignancies, autoimmune diseases, and endocrine abnormalities, and could also be induced by certain drugs including gold and bucillamine ${ }^{1,2}$.

A previously healthy 60-year-old man presented to our clinic with 1 year of discoloration of the nails and half a year of polyarthralgia. Physical examination revealed the presence of dystrophic nails characterized by thickened nail plates with yellowish-white discoloration and an increased transverse curvature involving all fingers nails with the cuticle and lunula disappearance (Figure 1A). Musculoskeletal system examination revealed the presence of tenderness and limitation of movement of the bilateral metacarpophalangeal and wrist joints. There was no sclerodactyly or telangiectasias.

Laboratory tests showed normal renal and hepatic function, and C-reactive protein level of $1.24 \mathrm{mg} / \mathrm{dL}$ (normal value $<0.3$ ). The patient was found to have high titers of rheumatoid factor $(425 \mathrm{IU} / \mathrm{mL})$ and anticyclic citrullinated peptide $(35 \mathrm{U} / \mathrm{mL})$. Test results for antinuclear antibody, antineutrophil cytoplasmic antibodies, antiphospholipid antibodies, several tumor markers, and thyroid hormone were negative or normal. Chest radiograph showed mild right-sided pleural effusion, which was evaluated by the pulmonologist. The patient was clinically diagnosed with yellow nail syndrome associated with rheumatoid arthritis. Treatment with sulfasalazine $1 \mathrm{~g}$ and vitamin E $300 \mathrm{mg}$ daily were initiated. One year after treatment, his arthritis and pleural effusion improved, and his nails returned to normal (Figure 1B).

This case should remind readers to consider systemic conditions including malignancies, autoimmune diseases, and endocrine abnormalities as underlying causes of yellow nail syndrome.

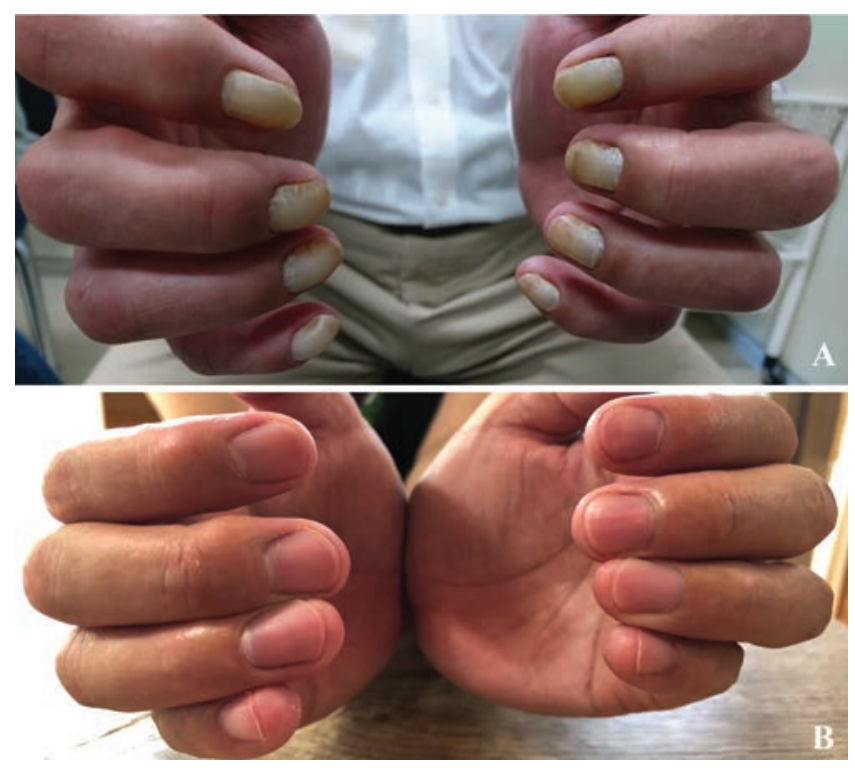

Figure 1. (A) Yellowish-white nail revealed in all fingers before treatment. (B) Yellowish-white nail returned to normal.

\section{REFERENCES}

1. Maldonado F, Ryu JH. Yellow nail syndrome. Curr Opin Pulm Med 2009;15:371-5.

2. David-Vaudey E, Jamard B, Hermant C, Cantagrel A. Yellow nail syndrome in rheumatoid arthritis: a drug-induced disease? Clin Rheumatol 2004;23:376-8. 